\title{
An Agent-Based Model of Avascular Tumor Growth
}

\author{
Ana Victoria Ponce Bobadilla ${ }^{1}$, René Doursat ${ }^{2}$ and François Amblard ${ }^{3}$ \\ ${ }^{1}$ Erasmus Mundus Master's in Complex Systems Science, \\ Graduate School, Ecole Polytechnique, Paris, France \\ ${ }^{2}$ BioEmergences Lab, CNRS (USR3695), Gif-sur-Yvette, France \\ ${ }^{3}$ Institut Curie, Paris, France \\ ana-victoria.ponce-bobadilla@polytechnique.edu
}

\begin{abstract}
We propose a simplified agent-based model of avascularized tumor. It involves a tissue in which blood vessels introduce nutrients that diffuse. Cells move, proliferate and die according to an individual quantity of "energy" and free space for their offspring. They can transition to a "cancerous" type and an intermediate "mutated" type, where they behave normally but can be affected by cancerous neighbors. We are interested in finding the key parameters that can lead a majority of cancerous cells to be replaced by normal ones. First, we give a brief overview of previous tumor growth models, especially in avascular tissues. Then, we describe in detail the agents and rules of our model, commenting on the choices made. Next, we conduct a parameter space exploration, varying in particular the influence of neighbors, the division probability and mutation probability. Results show a marked phase transition between domains of high cancerous cell density and high mutated cell density. We also analyze the importance of certain rules in our model by "rule knockout" and find that energy-dependency of division and space for offspring are essential, while type-specific division probabilities are not. Finally, we discuss the overall relevance of the model and possible future improvements.
\end{abstract}

\section{Introduction}

According to the World Health Organization, cancer is among the leading causes of death worldwide, with 8.2 million fatalities in 2012 (Stewart and Wild, 2014). Mathematical models are being created to help understand the underlying mechanisms of tumor growth, with the potential to create a framework for simulations and virtual experiments. This should enable scientists to observe the effects of different treatments more efficiently, and improve them or suggest new ones (Roose et al., 2007).

Cancer can generally be defined as the uncontrolled growth and spread of cells, also referred to as "malignant" tumors and "neoplasms". There are more than 100 types of cancers: lung, liver, stomach, colorectal and breast cancers are among the most lethal (Stewart and Wild, 2014). However, they all have certain characteristics in common: the tumor mass typically grows beyond its usual boundaries, invades neighboring parts of the body, then spreads out to remote organs.
It is still debated how exactly cancerous behavior is initiated. The general consensus is that several gene mutations are required to turn a normal cell into a cancer cell. The factors that trigger these mutations are largely unknown, but are thought to include both environmental and hereditary properties (Roose et al., 2007).

Once tumor cells have started to appear, tumor growth goes through three different stages:

- Avascular growth: This stage is characterized by the proliferation of cancer cells. The tumor forms a solid mass that expands by mitosis, depending largely on available nutrients. There is no invasion of healthy tissue yet. As nutrients deplete, the cancer cells die (necrosis) and their accumulation creates a necrotic core in the center of the tumor. During this stage, the tumor tends to adopt a spherical shape in which outer-perimeter cells continue to proliferate, while cells in the middle are in a resting state (quiescent). As necrosis and proliferation balance each other, the tumor reaches a limit size (approximately 1-3 $\mathrm{mm}$ in diameter).

- Tumor-induced angiogenesis: In this stage, cells from the avascular tumor mass induce modifications in the existing vascular structure to create new blood vessels that can sustain them. The tumor is able to overcome its limit size, grow much faster and invade the surrounding tissue.

- Vascular growth and invasive tumor: This is the most complex stage. The tumor does not form a solid mass anymore and becomes diffusive. Cancer cells can feed on nutrients beyond their immediate vicinity. The formation of necrotic regions becomes much more complex. Tumors do not have a limit size and can grow indefinitely.

In this work, we focus on the avascular stage of the tumor and the factors that can provoke its initial growth. We build a simplified model that takes into account key mechanisms identified through a selective review of previous models. We investigate how the model parameters affect the proportion of cancerous cells. Are there any critical values controlling a transition of the tumor from a majority of cancerous cells to a majority of normal ones? 


\section{Previous Work}

There is a large number of models of tumor growth, covering a great diversity of scales and questions. Byrne (2010) provides a timeline of the most representative models for each stage of tumor growth. Our own brief summary, focusing on the first stage, will not attempt to give an overview but rather highlight rules and parameters deemed important by the literature. An extensive review of models focusing on avascular tumor growth is given by Roose et al. (2007).

Most models fall into two broad categories: continuиm mathematical models, which are based on partial differential equations and spatial averages; and discrete cell population models, which involve individual cell-cell interactions.

\section{Continuum Cell Population Models}

These models focus on the relationships between cell density and chemical species that provide nutrients. Typically, they consist of reaction-diffusion and convection equations.

The earliest spatiotemporal and biomechanical models of avascular tumor growth construed the tumor as a 3D "multicellular spheroid" (MCS; Greenspan 1972). Tumor growth was regulated by a single diffusible chemical (oxygen or glucose), supplied externally. The resulting chemical distribution was then taken as a predictor of the underlying spheroid structure, comprising regions of cell proliferation, quiescence and necrosis. However, several simplifications gave these models little applicability: spheroids were assumed to grow radially and symmetrically, there was a single population of cells, and stochastic effects were ignored.

Several modifications and extensions were later brought to the MCS model: relaxing the assumption of radial symmetry, distinguishing different cell populations within the spheroid, and introducing cell movement and pressure (Araujo and McElwain, 2004). One of the most representative works (Casciari et al., 1992) considered a spherical tumor and the effects of certain chemical substances (oxygen, glucose, lactate, and carbon dioxide; bicarbonate, chloride, and hydrogen ions) on cell growth and metabolism. The basic principle at play here is that growth can be limited by chemical diffusion and nutrient consumption. This model also takes into account changes in cell proliferation rate within different chemical environments, and cell movement derived from a law of mass conservation.

Continuum models share several features: they do not distinguish between individual cells, they see tumors as continuous masses, stochastic effects are usually neglected, and subcellular phenomena are ignored. A good review of continuum models and techniques to analyze treatments can be found in (Perthame, 2014).

\section{Discrete Cell Population Models}

This category relies on various techniques, such as cellular automata (CA), lattice Boltzmann methods, agent-based modeling, extended Potts, and stochastic approaches (Roose et al., 2007). In all cases, cell state is generally characterized by a multidimensional variable $\mathbf{w}=\{x, v, u\}$, where $x$ is the position of the cell, $v$ its velocity, and $u$ its biological state, which may include its phase in the cell cycle, its interactions with the local chemical environment, and so on.

One of the first discrete models, proposed by Düchting and Vogelsaenger (1985), considered a complex cell cycle model in 3D. Another important study by Qi et al. (1993) used CA rules to reproduce the Gompertz law of cancer growth. Kansal et al. (2000) developed a 3D CA model that did not explicitly include nutrients or mechanical interactions but rather considered proliferation and death rates to be functions of position. "Cellular Potts" approaches, where each biological cell is made up of several lattice points, can also take into account cell membrane tension, cell-cell and cell-matrix adhesion, and chemotaxis (Turner and Sherratt, 2002). Recent years have seen a rise in "hybrid" models, which attempt to combine continuum equations for nutrient concentrations and CA models of cell cycle and migration (Trucu and Chaplain, 2014).

\section{Model}

We propose a new model of avascular tumor in its initial stages. The underlying space is a $2 \mathrm{D}$ tissue where nutrients are diffusing (Fig. 1). Cells can mutate and become cancerous with a certain probability. They move, proliferate, and die according to the local amount of nutrients, the free space for their offspring, and an internal "energy" that increases with nutrients. Cell positions and cell-cell interactions are constrained to a discrete $50 \times 50$ lattice. Cells are represented by circles inside square patches, and are initially assigned a certain position, energy level, and one of three possible types: "normal" (N), "mutated" (M) or "cancerous" (C). A patch can be occupied by one cell only. A few scattered patches coded in red represent blood vessels and can also be occupied by one cell. Despite the presence of blood vessels, this remains a stage- 1 avascular tumor simulation since we are not including stage- 2 angiogenetic mechanisms. Setup and update rules are explained below.

\section{Agents and Variables}

There are two agents in the model: cells and patches. An M cell behaves like an $\mathrm{N}$ cell with the difference that it can be affected by its $\mathrm{C}$ neighbors. The simulation begins with an initial number of cells, $n$, and a fixed number of blood-vessel patches, $b$. Each cell $i=1, \ldots, n$ is assigned a state vector $\mathbf{w}_{i}=\left(x_{i}, y_{i}, e_{i}, \tau_{i}, x_{i}^{\prime}, y_{i}^{\prime}\right)$ representing its position on the grid, its energy level in $[0,1]$, its type in $\{\mathrm{N}, \mathrm{M}, \mathrm{C}\}$, and free space for its offspring, which can be any unoccupied patch inside a von Neumann domain (4 nearest neighbors) around $\mathrm{N}$ and $\mathrm{M}$ cells, or a Moore domain (8 nearest neighbors) around $\mathrm{C}$ cells. Each patch $j$ on the lattice, whether occupied or not by a cell, is assigned a nutrient level $\nu_{j}$ and a type that can be either "tissue" (T) or "blood vessel" (B). 


\begin{tabular}{|l|c|c|}
\hline Parameter & Symbol & Value \\
\hline Initial number of N cells & $n$ & 200 \\
\hline Fixed number of B patches & $b$ & 30 \\
\hline Diffusion weight & $d$ & 0.8 \\
\hline Unit of nutrient consumption & $c$ & 0.02 \\
\hline Division energy of N, M cells & $E_{N}$ & 0.7 \\
\hline Division energy of C cells & $E_{C}$ & 0.6 \\
\hline Division probability of N, M cells & $p_{N}$ & 0.7 \\
\hline Division probability of C cells & $p_{C}$ & 0.8 \\
\hline Mutation probability in $\mathrm{N} \rightarrow \mathbf{M} \rightarrow \mathbf{C}$ & $m$ & 0.05 \\
\hline Influence of N, M neighb. on C $\rightarrow \mathbf{M}$ & $\epsilon_{N}$ & 0.8 \\
\hline Influence of C neighb. on $\mathbf{M} \rightarrow \mathbf{C}$ & $\epsilon_{C}$ & 0.4 \\
\hline
\end{tabular}

Table 1: Parameters of the model and their standard values.

\section{Setup}

First, the parameters of the model (Table 1) are given initial or constant values. Next, each patch is assigned a random nutrient level $\nu_{j}$ uniformly drawn in $[0,1]$ and a corresponding green-scale color (Fig. 1). Then, $n$ cells are created and placed randomly on the patches. All cells are initialized to type $\mathrm{N}$ and assigned a random energy value $e_{i}$ uniformly drawn in $[0,1]$. Finally, $b$ blood-vessel patches are randomly created, possibly overlapping with cells, and colored in red.

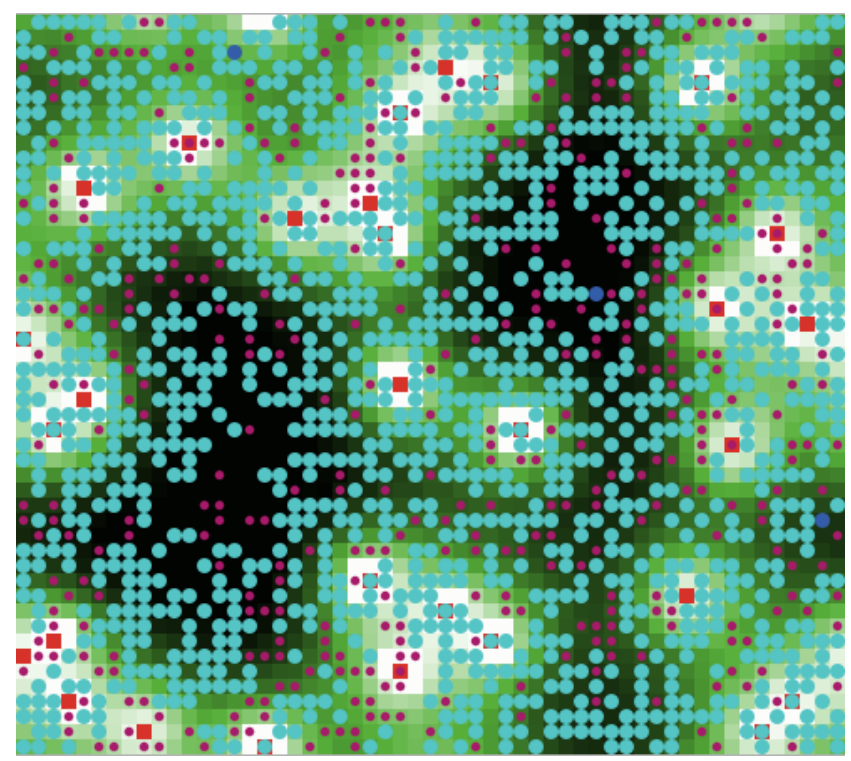

Figure 1: Screenshot of the model after 100 time steps under the parameter values of Table $1 . \mathrm{N}, \mathrm{M}$ and $\mathrm{C}$ cells are colored in blue, cyan and magenta respectively. Patches are in greenscale according to nutrient levels (white for $\nu_{j}=1$, black for $0)$. Here, the tissue contains a large majority of M cells.

\section{Update Rules}

At each time step, three rules are applied: cell update, patch update and type update (Fig. 2).
Cell update First, each cell $i$ checks if there are nutrients in the patch $j$ that it occupies, i.e. whether $\nu_{j}>0$. If not, the cell dies with probability $1-e_{i}$, or if it stays alive, does nothing. If there are nutrients, it consumes a fixed amount $c$, up to the available quantity $\nu_{j}$, which increases its energy level accordingly: $e_{i} \leftarrow e_{i}+\min \left(c, \nu_{j}\right)$, while the nutrient level of the patch is decreased by the same quantity: $\nu_{j} \leftarrow$ $\nu_{j}-\min \left(c, \nu_{j}\right)$. Then, if the cell is cancerous (C), it looks for free space in its 8-patch neighborhood (including blood vessels) and whether it has enough energy to divide, i.e. $e_{i}>$ $E_{C}$. This leads to four different scenarios:

- Free space, enough nutrients: The $\mathrm{C}$ cell divides with probability $e_{i} * p_{C}$ and, if it divides, reduces its energy by the cost of division: $e_{i} \leftarrow e_{i}-E_{C}$. Then, it splits this remaining energy into two, keeping one half $e_{i} / 2$ and giving the other half to its daughter.

- Free space, not enough nutrients: The C cell moves randomly to one of the unoccupied 8 neighboring patches (including on top of a blood vessel).

- No free space, enough nutrients: The C cell waits.

- No free space, not enough nutrients: The $\mathrm{C}$ cell dies with probability $1-e_{i}$, or does nothing.

The same rules apply to noncancerous cell types $\mathrm{N}$ and $\mathrm{M}$, except that they use a 4-patch neighborhood, a division energy $E_{N}$, and a division probability $e_{i} * p_{N}$. Moreover, they wait instead of moving in the second case.

Patch update If the patch $j$ is a blood vessel, then it replenishes its 8 nearest neighbors $k$ with maximum nutrient levels: $\nu_{k} \leftarrow 1$. After that, nutrients diffuse around all patches with a "diffusion weight" $d \in[0,1]$. It means that a portion $d$ of each patch's nutrient level is equally distributed over its 8 nearest neighbors: $\nu_{k} \leftarrow \nu_{k}+(d / 8) * \nu_{j}$.

Type update (i) If a cell is type N, it can turn into type $M$ with fixed probability $m$, then it waits until the next time step. (ii) If it is type $M$, it can turn into type $C$ with the same probability $m$. Then, if it is still type $\mathrm{M}$, it can turn again into type $\mathrm{C}$, this time with probability $\epsilon_{C} * n_{i}^{C} / n_{i}$, where $n_{i}$ is the number of neighboring patches (among 8) occupied by a cell and $n_{i}^{C}$ is the number of type-C neighbors. After that, it waits. (iii) If a cell is type $\mathrm{C}$, it can revert to type $\mathrm{M}$ with probability $\epsilon_{N} * n_{i}^{N} / n_{i}$, where $n_{i}^{N}$ is the number of type-N and type-M neighbors counted together. Coefficients $\epsilon_{N}$ and $\epsilon_{C}$ are referred to as the "influence of neighbors".

\section{Rationale}

Variables and rules are chosen to reflect a realistic, yet simplified, view of tumor development. Ignoring the detailed biochemical activity inside each cell, our intention is to focus on a few key features that affect tumor growth. To represent the competitive advantage that cancerous cells have 


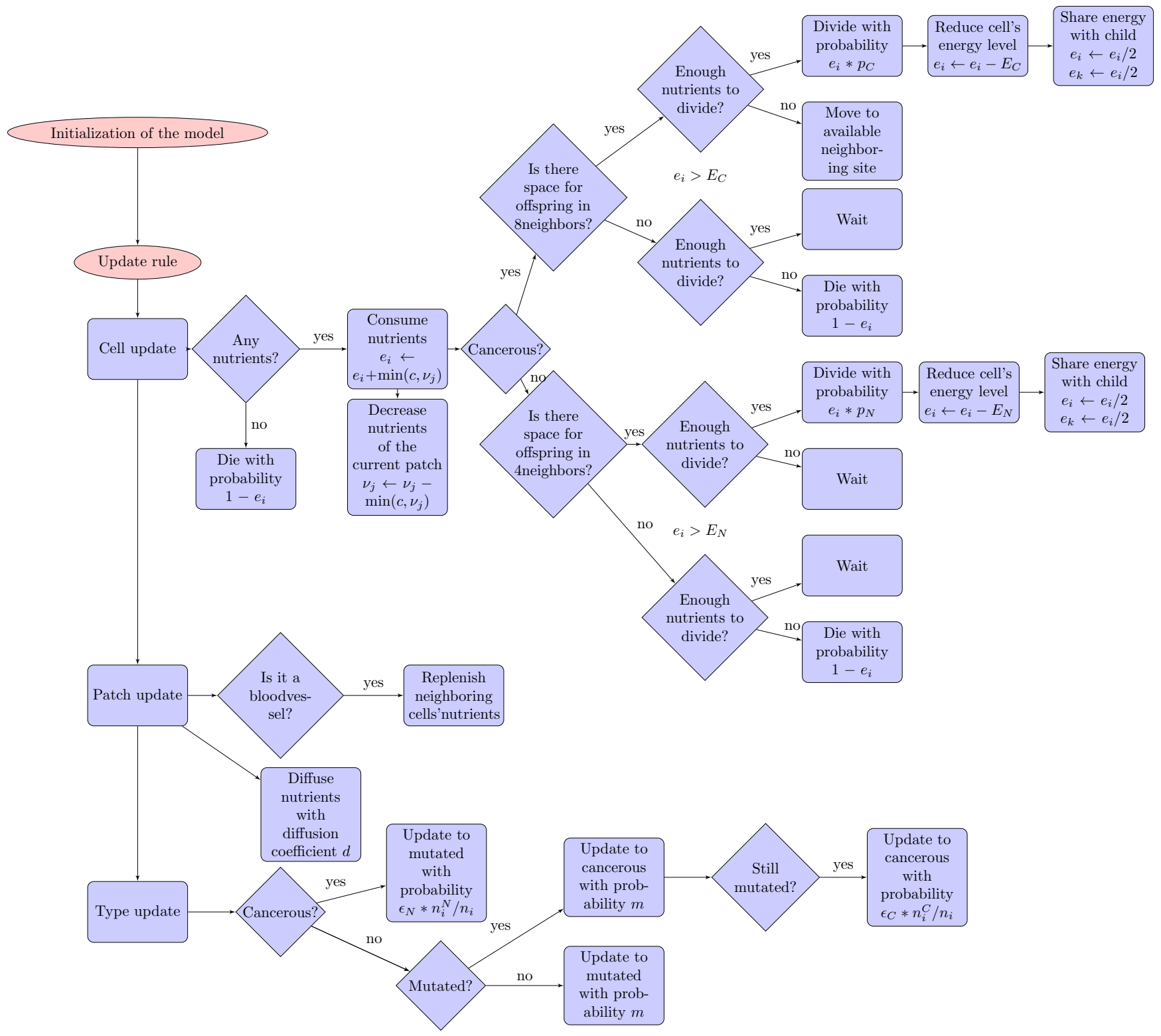

Figure 2: Flowchart of the initialization and update rules.

over noncancerous cells, we set their division probability to a larger value $\left(p_{C}>p_{N}\right)$, their corresponding energy cost to a smaller value $\left(E_{C}<E_{N}\right)$, and the space that their offspring can potentially occupy to 8 patches instead of 4 . When nutrients are low, $\mathrm{C}$ cells can also move to a free patch, as opposed to just waiting like $\mathrm{N}$ and $\mathrm{M}$ types.

Our introduction of an abstract $\mathrm{M}$ type is meant to provide an intermediate type between $\mathrm{N}$ and $\mathrm{C}$ cells. The idea is that $\mathrm{M}$ cells are "carriers of the disease" but still behave like normal cells. They represent the possibility for cancerous cells to revert to a certain form of normalcy (observed experimentally), yet without fully returning to the original $\mathrm{N}$ type, so they are still susceptible to their neighbors' influence. One could say that mutated cells are similar to the hypothesis of "dormant" cells in avascular tumors (Udagawa et al., 2002).

We also include the energy as a factor in the actual probabilities of division, $e_{i} * p_{N}$ and $e_{i} * p_{C}$, to increase the spread of cells that have more energy, or had enough energy for a while and were only waiting for neighboring space to open up. Finally, concerning the influence of neighbors, it is important to note that if $\epsilon_{N}=1$ and $\epsilon_{C}=0$, there would be only mutated cells at the end of the simulation, whereas in the opposite case, there would be only cancerous cells. Therefore these two parameters must be carefully adjusted to represent a realistic situation. This is one of the topics of the parametric study that follows. 


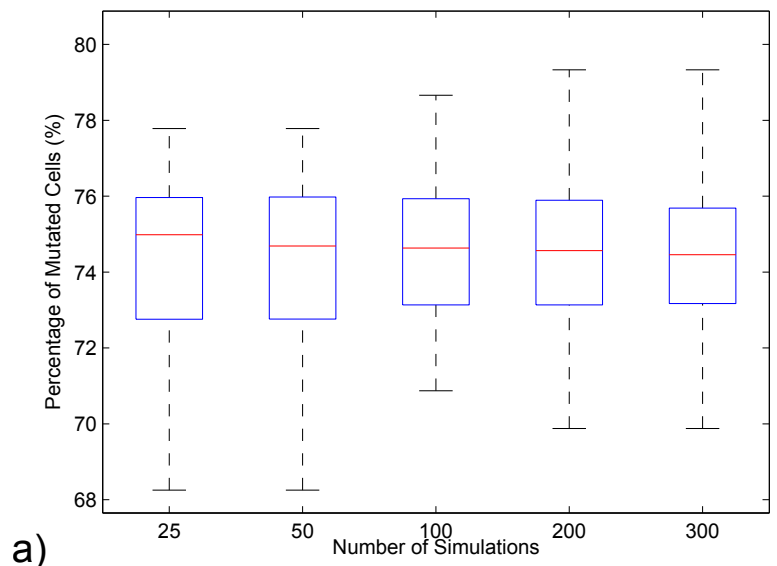

b)

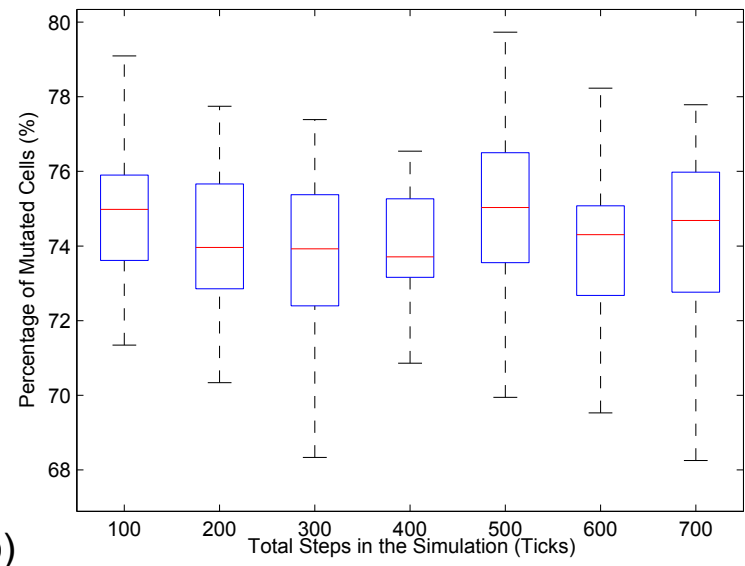

Figure 3: Sensitivity analysis of the model. (a) Average density of mutated cells, $r_{M}=n_{M} / n$, calculated over various numbers of simulations lasting 700 time steps each. (b) Same average density calculated over 100 simulations lasting various numbers of time steps. All other parameters are set to the standard values of Table 1 . The mean and standard deviation of $r_{M}$ change only little, at around $\overline{r_{M}} \approx 74.5 \%$ and $\Delta r_{M} \approx 2.5 \%$, indicating that the behavior of the model is consistent across experiments.

\section{Results}

\section{Measurements}

To analyze the dynamics of the model, we measure the overall densities and "clustered" densities of normal, mutated and cancerous cells. An $\mathrm{N}$ cell is said to belong to a cluster if there are at least 5 other $\mathrm{N}$ cells in its 8-patch neighborhood (same for $\mathrm{M}$ and $\mathrm{C}$ cells).

\section{Sensitivity Analysis}

Since the model is stochastic, we must evaluate the number of simulations $S$ and the number of time steps per simulation $T$ needed to conduct a parameter space exploration that is sufficiently representative of the generic model behavior. To carry out this sensitivity analysis, we use the parameters of Table 1 and calculate the overall density of $\mathrm{M}$ cells, $r_{M}=n_{M} / n$. Setting $T=700$ under various numbers of simulations $S=25, \ldots, 300$ (Fig. 3a), we observe that the range of $r_{M}$ (mean and variance) is about constant for $S \geq 100$. Conversely, setting $S=100$ under various durations $T=100, \ldots, 700$ (Fig. $3 b$ ) shows that $r_{M}$ tends to stabilize for $T \geq 500$. Therefore, we conclude that the behavior our model is robust and adopt $S=100$ and $T=500$ to keep the computing cost reasonable in the rest of the study, since the distributions of $r_{M}$ are similar above these values.

\section{Parameter Space Exploration}

To explore the dynamical regimes of the model, we select the following dimensions of parameter space, expected to have an important impact on the outcome: the influence of neighbors, $\epsilon_{N}$ and $\epsilon_{C}$; the division probability of noncancerous cells, $p_{N}$; and the mutation probability, $m$. In each case, a pair of values or a single value is varied, while all other parameters are kept at the standard settings of Table 1.
Influence of neighbors We systematically vary $\epsilon_{N}$ and $\epsilon_{C}$ in the interval $[0,1]$ by increments of 0.1 , for a total of $11 \times 11$ experimental points, and plot the mean and standard deviation of the density of $\mathrm{M}$ cells and $\mathrm{C}$ cells (Fig. 4). The mean density of $\mathrm{N}$ cells consistently remains under $3 \%$ and is not shown. This reveals a phase transition from a majority of $\mathrm{C}$ cells to a majority of $\mathrm{M}$ cells along a boundary line roughly at $\epsilon_{N}=\epsilon_{C}+0.2$. Therefore, to push a cancerous tissue back into a majority of $\mathrm{M}$ cells, the mutating influence of $\mathrm{N}$ and $\mathrm{M}$ cells has to be significantly greater than that of $\mathrm{C}$ cells.

Division probability We choose two points in the high M-cell density domain of the neighbors' influence space (Fig. 4a): $A=\left(\epsilon_{N}, \epsilon_{C}\right)=(0.8,0.1)$ and $B=(0.8,0.4)$ (the standard values), and vary the division probability of $\mathrm{N}$ cells $p_{N}$ in $[0,0.7]$ by increments of 0.1 . We observe that in point $A, p_{N}$ does not have much effect on the dynamics in the model, which invariably ends with many more $\mathrm{M}$ cells than $\mathrm{C}$ cells, about $85 \%$ vs. $15 \%$, while $\mathrm{C}$ cells remain isolated (Fig. 5a,c). In point $B$, by contrast, pulling $p_{N}$ below 0.2 makes the densities of $\mathrm{M}$ and $\mathrm{C}$ cells converge to comparable values, about $50 \%$ overall and $15-20 \%$ in clusters (Fig. 5b,d). It means that to stay outside the high cancerous density domain, $\mathrm{N}$ and $\mathrm{M}$ cells should have a greater mutating influence than $\mathrm{C}$ cells on their neighbors (previous conclusion), or $\mathrm{N}$ and $\mathrm{M}$ cells should divide reasonably fast.

Random mutation probability With the standard parameters of Table 1, we vary the cancerous neighbors' influence $\epsilon_{C}$ under three different basic $\mathrm{N} \rightarrow \mathrm{M} \rightarrow \mathrm{C}$ mutation probabilities. Under the standard low value $m=0.05$, we find again a transition between high M-cell and high C-cell density domains at $\epsilon_{C} \approx 0.6$ (Fig. 6a). Under a higher value $m=0.15$, the transition happens earlier at $\epsilon_{C} \approx 0.2$ (Fig. 6b). Under very high mutation rate $m=0.25$, the neighbors' influence 

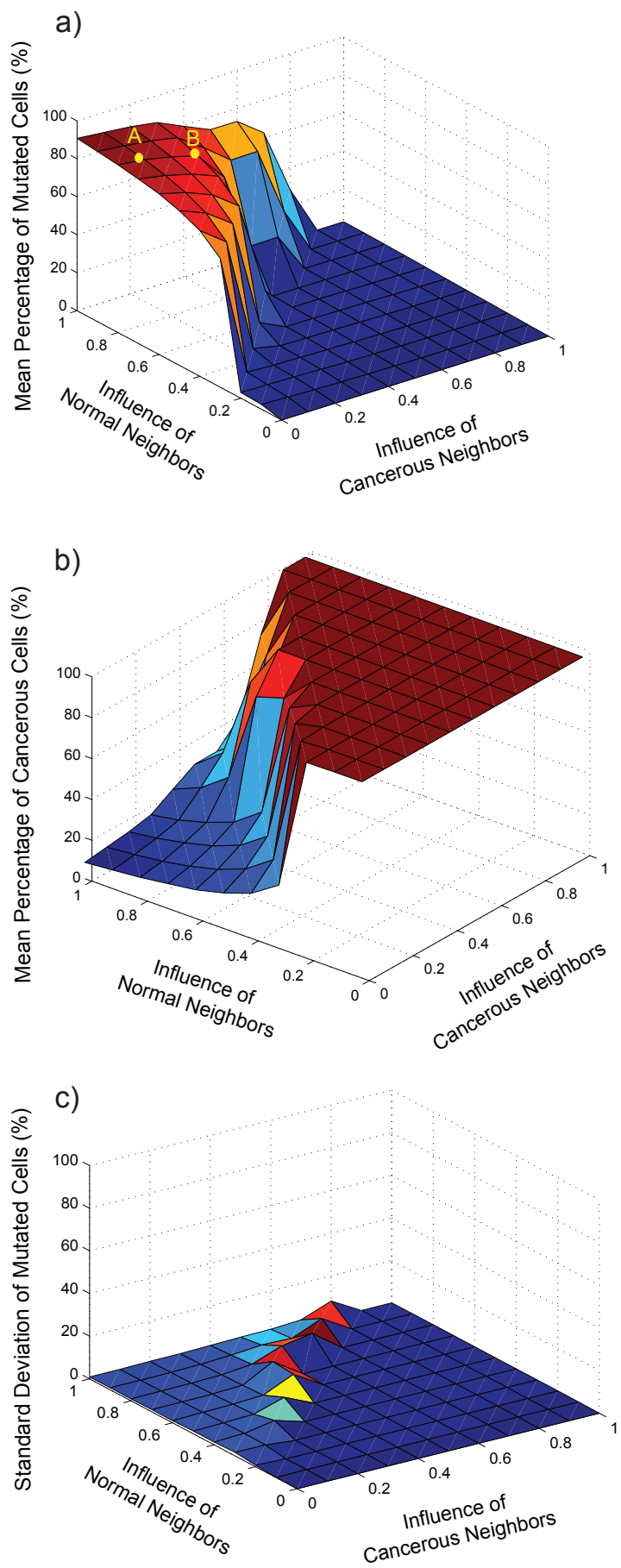

Figure 4: Exploring the influence of neighbors. Both mutating factors, $\epsilon_{N}$ and $\epsilon_{C}$, are varied in $[0,1]$ while the other parameters are as in Table 1. (a) Mean density of mutated cells, $\overline{r_{M}}$. (b) Mean density of cancerous cells, $\overline{r_{C}}$. (c) Standard deviation of density of M cells, $\Delta r_{M}$ (same as $\Delta r_{C}$, not shown here). All graphs exhibit a clear phase transition along a boundary line roughly at $\epsilon_{N}=\epsilon_{C}+0.2$. Parametric point $B=\left(\epsilon_{N}, \epsilon_{C}\right)=(0.8,0.4)$ in the high M-cell density domain is the standard. The other point, $A=(0.8,0.1)$, will be used when varying the division probability $p_{N}$ (Fig. 5). is ignored by the system: almost all cells are cancerous and no transition back to a mutated state is possible. (Fig. 6c).

\section{Importance of Rules}

We also analyze the importance of certain rules by measuring the effects of "rule knockout" on our model: (i) removing energy-dependency from division probabilities and setting them to constant rates $p_{N}$ and $p_{C}$; (ii) conversely, removing type-specificity from division probabilities and setting them to the energy level $e_{i}$ for all cells; (iii) finally, removing type-specificity from division space and giving all cells the same 8-patch Moore neighborhood to spawn offspring. In each scenario, the neighbors' influence parameter space $\left(\epsilon_{N}, \epsilon_{C}\right) \in[0,1]^{2}$ is explored again and the transition area between high M-cell and high C-cell density domains is estimated and plotted in 2D (Fig. 7). A parametric point is considered to lie inside the transition area if the variance of the M-cell density, $\left(\Delta r_{M}\right)^{2}$, is greater than the mean variance over all 121 points. We observe that scenarios (i) and (iii) provoke a significant displacement of the transition area (Fig. 7b,d), whereas scenario (ii) leaves it almost unchanged (Fig. 7c). Therefore, we conclude that energy-dependency of division and offspring space are key mechanisms, while type-specificity of division is not.

\section{Discussion}

We presented a simple agent-based model of tumor growth able to give rise to two different situations: one where the majority of cells is cancerous and another one where it is mutated. We showed the outcomes of varying the influence of neighbors, division probabilities and mutation probability. A constant feature of the system's behavior is that most normal cells disappear and only a competition between mutated and cancerous cells remains (Fig. 1). The biological conclusion is that, in the end, all normal cells become influenced by the type of their neighbors. We could expect normal cells to survive longer, however the rules and standard parameter values of our model give a clear advantage to cancerous cells across all dimensions (more space for the offspring, less division energy, higher probability of dividing) and lead to a rapid depletion of normal cells.

Our exploration of neighbors' influence parameter space revealed a phase transition area, inside which the standard deviation of cell densities is clearly positive, i.e. the outcomes of simulations vary significantly more (Fig. 4). This implies the existence of two types of parametric points in the model: ones such as $A$ which are far away from the phase transition and create a stable system, and ones such as $B$ which are close to the phase transition and create instability. This was illustrated by varying the division probability in point $B$ and showing that the density of mutated cells could drop below that of cancerous cells (Fig. 5b,d). Another transition between highly mutated and highly cancerous states was found along the mutation probability axis at 
a)

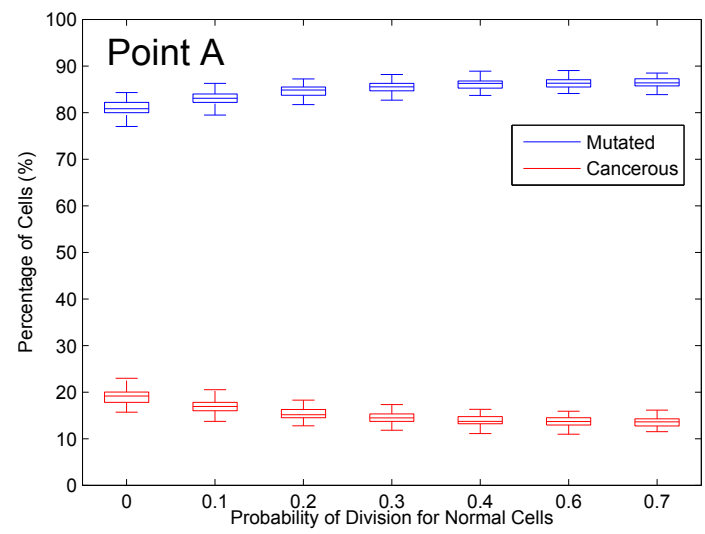

c)

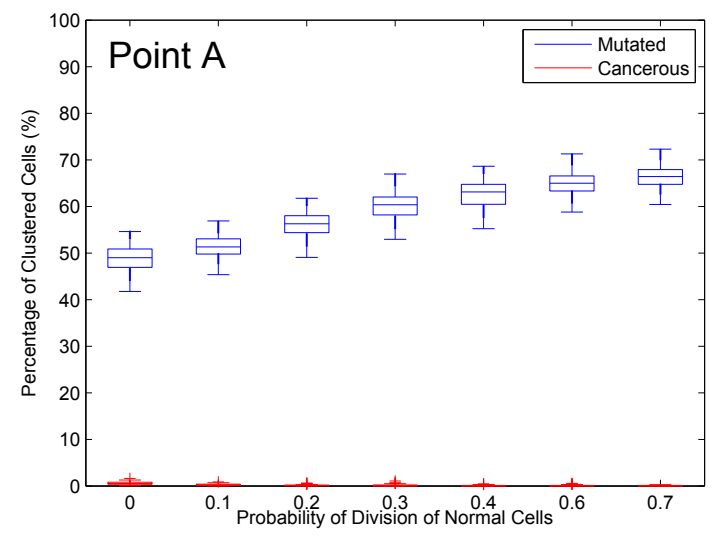

b)

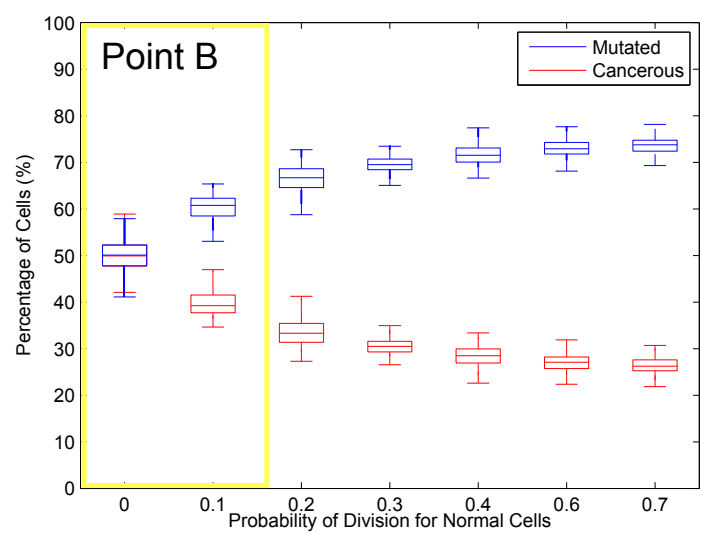

d)

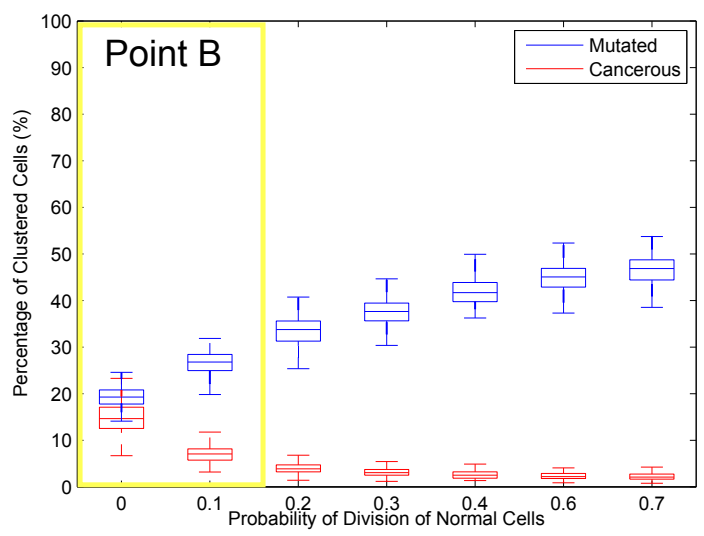

Figure 5: Exploring the division of noncancerous cells. The probability $p_{N}$ is varied while other parameters are as in Table 1, except the pair $\left(\epsilon_{N}, \epsilon_{C}\right)$ which is set to point $A$ or point $B$ as in Fig. 4a. (a,b) Average overall densities of mutated cells (in blue) and cancerous cells (in red). (c,d) Average densities of clustered M and C cells. (a,c) Parametric conditions $A: p_{N}$ has little effect on the outcome, characterized by a significant majority of M cells and isolated C cells. (b,d) Standard parametric conditions $B$ : low values of $p_{N}$ (highlighted in yellow) lead to comparable numbers of $\mathrm{M}$ and $\mathrm{C}$ cells.

around $20 \%$, above which only $\mathrm{C}$ cells remained (Fig. 6).

By varying these parameters, we examined both internal and external factors of tumor growth. In future work, the diffusion weight $d$ will be another important parameter to explore, in order to check whether growth can be limited by diffusion as it was the case in earlier models of avascular tumors. Finally, by analyzing the effect of rule knockout on the transition curve we were able to assess the significance or neutrality of certain rules in our model.

Compared to our work, other agent-based models in the literature generally contain more complex and realistic descriptions of cellular agents and tumor environment (review in Wang et al., 2015). They strive to take into account multiple phenotypic features such as molecular signaling, cellular metabolism and other mutation-induced changes (e.g. Ramis-Conde et al., 2008). By contrast, our goal was to identify a minimal set of rules enabling behaviors typical of avascular tumor growth: proliferation in nutrient-rich re- gions, limitation in size, and phase transitions. This also allowed us to conduct a parameter space exploration along a few dimensions and assess the effects of varying key values.

Naturally, our model can be improved in several ways. For example, in order to minimize the processing time, we assumed that nutrients diffused at constant speed regardless of patch occupancy. In reality, proteins, lipids and other particles undergo anomalous diffusion due to molecular crowding (Banks and Fradin, 2005). Second, instead of setting the parameters to empirical values appropriate for the numerical simulations, one could try to match them to real imaging datasets and measurements from specific tumors, for example via a "fitness" function evaluating the realism (overlap) between the digital and the physical object.

In sum, we tried to describe the dynamics of the initial stages of avascular tumor growth using only key principles and concepts without including chemical or mechanical details. Future extensions of this model should take into ac- 

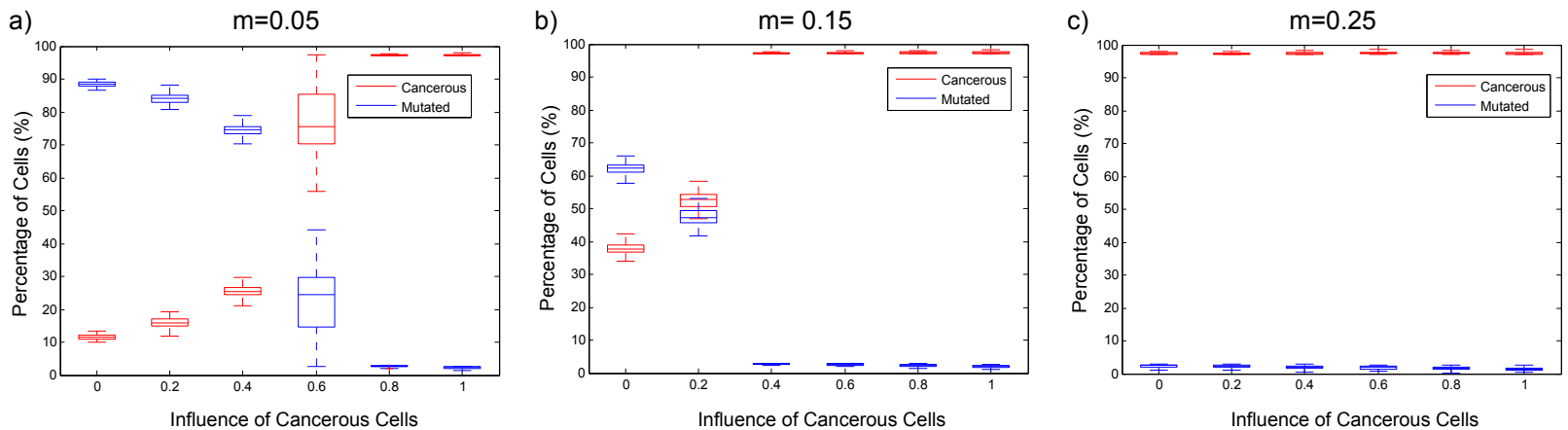

Figure 6: Exploring the mutation probability $m$. The cancerous neighbors' influence $\epsilon_{C}$ is varied while other parameters remain as in Table 1. (a) At the standard, low $m$ value, we obtain the same transition as Fig. 4. (b,c) At higher $m$ values, the transition shifts in favor of all-cancerous end states.

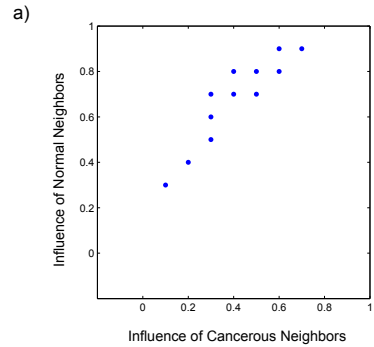

c)

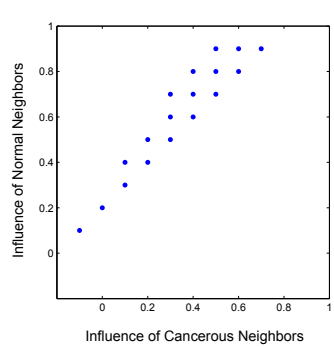

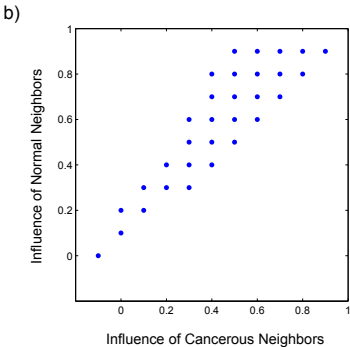

d)

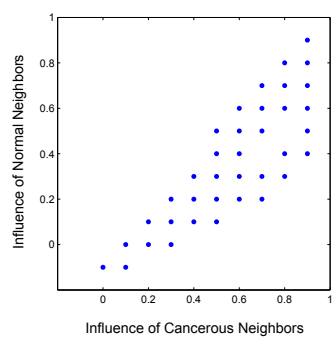

Figure 7: Importance of rules. The transition curve in $\left(\epsilon_{N}, \epsilon_{C}\right)$ space can be more or less shifted in different versions of the model. (a) Standard rules: curve as in Fig. 4. (b) No dependence on energy in the division probabilities $\rightarrow$ significant shift. (c) No distinction between types in the division probabilities $\rightarrow$ no significant shift. (d) More neighborhood space for noncancerous cells $\rightarrow$ significant shift.

count both aspects as well as the cell cycle. Moreover, they should address the possibility of fitting the parameters to clinical data about the delimitation of the proliferating region, the location of the phase transition, and the limit size of the avascular tumor.

\section{References}

Araujo, R. and McElwain, D. (2004). A history of the study of solid tumour growth. Bull. Math. Biol., 66(5):1039-1091.

Banks, D. S. and Fradin, C. (2005). Anomalous diffusion of proteins due to molecular crowding. Biophysical Journal, 89(5):2960-2971.

Byrne, H. M. (2010). Dissecting cancer through mathematics: from the cell to the animal model. Nature Reviews Cancer, 10(3):221-230.

Casciari, J., Sotirchos, S., and Sutherland, R. (1992). Mathematical modelling of microenvironment and growth in emt6/ro multicellular tumour spheroids. Cell Proliferation, 25(1):1-22.

Düchting, W. and Vogelsaenger, T. (1985). Recent progress in modelling and simulation of three-dimensional tumor growth and treatment. Biosys., 18(1):79-91.

Greenspan, H. (1972). Models for the growth of a solid tumor by diffusion. Stud. Appl. Math, 51(4):317-340.

Kansal, A., Torquato, S., Chiocca, E., and Deisboeck, T. (2000). Emergence of a subpopulation in a computational model of tumor growth. J. Theo. Biol. 207(3):431-441

Perthame, B. (2014). Some mathematical aspects of tumor growth and therapy. In ICM 2014.

Qi, A.-S., Zheng, X., Du, C.-Y., and An, B.-S. (1993). A cellular automaton model of cancerous growth. J. Theo. Biol., 161(1):1-12.

Ramis-Conde, I., Drasdo, D., Anderson, A. R., and Chaplain, M. A. (2008). Modeling the influence of the e-cadherin- $\beta$-catenin pathway in cancer cell invasion: a multiscale approach. Biophysical journal, 95(1):155-165.

Roose, T., Chapman, S. J., and Maini, P. K. (2007). Mathematical models of avascular tumor growth. Siam Review, 49(2):179-208.

Stewart, B. W. and Wild, C. P. (2014). World cancer report 2014. IARC Press, International Agency for Research on Cancer.

Trucu, D. and Chaplain, M. A. (2014). Multiscale analysis and modelling for cancer growth and development. In Managing Complexity, Reducing Perplexity, pages 45-53. Springer.

Turner, S. and Sherratt, J. A. (2002). Intercellular adhesion and cancer invasion. $J$. Theo. Biol., 216(1):85-100.

Udagawa, T., Fernandez, A., Achilles, E.-G., Fokman, J., and DAmato, R. J. (2002). Persistence of microscopic human cancers in mice: alterations in the angiogenic balance accompanies loss of tumor dormancy. The FASEB journal, 16(11):1361-1370

Wang, Z., Butner, J. D., Kerketta, R., Cristini, V., and Deisboeck, T. S. (2015). Simulating cancer growth with multiscale agent-based modeling. Seminars in Cancer Biology, 30:70-78. 